\title{
Intercellular calcium wave propagation in linear and circuit-like bone cell networks
}

\author{
By Bo HuO ${ }^{1,2, \dagger}$, XIN L. Lu ${ }^{1, \dagger}$ AND X. Edward GuO ${ }^{1, *}$ \\ ${ }^{1}$ Bone Bioengineering Laboratory, Department of Biomedical Engineering, \\ University of Columbia, 351 Engineering Terrace, PO Box 8904, \\ 1210 Amsterdan Avenue, New York, NY 10027, USA \\ ${ }^{2}$ Centre for Biomechanics and Bioengineering, Key Laboratory of Microgravity, \\ Institute of Mechanics, Chinese Academy of Sciences, Beijing 100190, \\ People's Republic of China
}

In the present study, the mechanism of intercellular calcium wave propagation in bone cell networks was identified. By using micro-contact printing and self-assembled monolayer technologies, two types of in vitro bone cell networks were constructed: open-ended linear chains and looped hexagonal networks with precisely controlled intercellular distances. Intracellular calcium responses of the cells were recorded and analysed when a single cell in the network was mechanically stimulated by nano-indentation. The looped cell network was shown to be more efficient than the linear pattern in transferring calcium signals from cell to cell. This phenomenon was further examined by pathway-inhibition studies. Intercellular calcium wave propagation was significantly impeded when extracellular adenosine triphosphate (ATP) in the medium was hydrolysed. Chemical uncoupling of gap junctions, however, did not significantly decrease the transferred distance of the calcium wave in the cell networks. Thus, it is extracellular ATP diffusion, rather than molecular transport through gap junctions, that dominantly mediates the transmission of mechanically elicited intercellular calcium waves in bone cells. The inhibition studies also demonstrated that the mechanical stimulation-induced calcium responses required extracellular calcium influx, whereas the ATP-elicited calcium wave relied on calcium release from the calcium store of the endoplasmic reticulum.

Keywords: osteoblast; AFM indentation; gap junction; ATP; calcium source

\section{Introduction}

Since Julius Wolff, a German anatomist, suggested the concept of mechanical stimulation-induced bone remodelling 110 years ago, numerous studies have been performed to clarify the mechanism of bone adaptation (Allori et al. 2008). With the understanding that it is the bone cells that detect the mechanical signals in situ and integrate these signals into appropriate changes in the

*Author for correspondence (ed.guo@columbia.edu).

${ }^{\dagger}$ These authors contributed equally to the work.

One contribution of 9 to a Theme Issue 'Multi-scale biothermal and biomechanical behaviours of biological materials'. 
bone architecture, modern scientists have focused on characterizing the mechanotransduction pathways in bone cells. The mechanism of how cells detect mechanical signals and how to propagate the signals to neighbouring bone cells yet remain unclear, but it was found that the fluctuation of intracellular calcium $\left(\left[\mathrm{Ca}^{2+}\right]_{\mathrm{i}}\right)$ concentration is one of the earliest responses in bone cells when they are under mechanical stimulation (Hung et al. 1995). Apart from being deposited in the mineralized bone matrix, the calcium ion $\left(\mathrm{Ca}^{2+}\right)$ is now recognized as an essential messenger in bone cells throughout their entire lifespan. Calcium signalling plays critical roles in regulating osteoclastogenesis (Kuroda et al. 2008), osteoblast proliferation and differentiation (Zayzafoon 2006), and the mechanobiology of osteocytes (Yellowley et al. 2000; Rubin et al. 2006). The mechanically induced $\left[\mathrm{Ca}^{2+}\right]_{\text {i }}$ responses can also propagate from cell to cell in bone tissue, which provides a critical mechanism for intercellular communication and coordination of cellular activities (Jorgensen et al. 1997, 2000; Carafoli 2002).

The calcium ions in the cytosol are dynamically regulated with those in intracellular stores (e.g. endoplasmic reticulum (ER) in bone cells) via gating mechanisms and with those in the extracellular solution through membrane ion channels and pumps. We and others have found that the elevation of cytosolic calcium has to be induced by the influx of extracellular calcium when a bone cell is mechanically stimulated (Batra et al. 2005; Huo et al. 2008). The calcium influx further leads to phospholipase C (PLC) synthesis of inositol 1,4,5-trisphosphate $\left(\mathrm{IP}_{3}\right)$ and triggers calcium release from $\mathrm{IP}_{3}$-sensitive intracellular calcium stores, such as from the ER (Berridge 1993). One mechanism by which bone cells propagate calcium signalling to other cells is the transfer of calcium and $\mathrm{IP}_{3}$ via gap-junctional intercellular communication (GJIC; Donahue et al. 1995; Jorgensen et al. 2000). Gap junctions are membranespanning channels that allow rapid (milliseconds) and direct transfer of small molecules $(<1 \mathrm{kDa})$, such as ionized calcium and inositol phosphates, from the cytosol of one cell to the cytosol of connected cells. Since abundant gap junctions exist between osteocytes and osteoblasts (Duncan \& Turner 1995; Yellowley et al. 2000), it has long been conjectured that GJIC is responsible for intercellular calcium propagation in bone cells. However, recent studies on glial cells (Guthrie et al. 1999), juxtaglomerular cells (Yao et al. 2003) and two osteoblastic cell lines (Jorgensen et al. 1997) have shown that intercellular calcium waves can be propagated between physically isolated cells without gap junction communication. In those cells, calcium waves appear to be mediated by the activation of purinergic receptors, presumably by secreted extracellular adenosine triphosphate (ATP). ATP can bind to the membrane-bound P2Y nucleotide receptors, resulting in activation of the $\mathrm{PLC}^{-\mathrm{IP}_{3}}$ pathway (Berridge et al. 2000; Iqbal \& Zaidi 2005). Thus, diffusion of extracellular ATP appears to be another powerful candidate for the mechanism behind intercellular calcium waves in bone cell networks.

In our previous studies (Guo et al. 2006), a two-dimensional patterned bone cell network with controlled intercellular gap junction connections and cellto-cell distance was successfully established by using micro-contact printing and self-assembled monolayer (SAM) technologies. Each cell in the grid network was connected with four neighbouring cells via functional gap junctions through uniform distances. This more physiologically relevant network normalizes the extracellular environment of each individual cell and provides inherent advantages 
to quantitatively characterize signal propagation between cells. We also studied the $\left[\mathrm{Ca}^{2+}\right]_{\mathrm{i}}$ oscillations in patterned MC3T3-E1 cell networks and the roles of ATP and gap junction pathways (Huo et al. 2008) under fluid shear stimulation. It was found that multiple $\left[\mathrm{Ca}^{2+}\right]_{\mathrm{i}}$ responses were significantly attenuated when purinergic receptors on the plasma membrane were inhibited, but the multiple responses were less affected when gap junctions were blocked. Moreover, the $\left[\mathrm{Ca}^{2+}\right]_{\mathrm{i}}$ oscillation induced by fluid flow relied on both extracellular calcium influx and the release of the ER calcium store.

In this study, we built a one-dimensional linear cell chain and employed nanoindentation on one single cell in the chain as mechanical stimulation. By analysing the calcium wave in the cell chain treated with different pathway inhibitors, the roles of GJIC and extracellular ATP diffusion in calcium wave propagation in bone cell networks were clarified. To verify the results, the calcium wave in a looped hexagonal cell network was also obtained and compared with those from the linear networks.

\section{Material and methods}

(a) Chemicals

Minimum essential alpha medium ( $\alpha$-MEM), calcium-free Dulbecco's modified eagle medium (DMEM), calcium-free Hank's balanced salt solution (HBSS) and DMSO were obtained from Invitrogen Corporation (Carlsbad, CA, USA). Foetal bovine serum (FBS), charcoal-stripped FBS, and penicillin/streptomycin $(\mathrm{P} / \mathrm{S})$ were purchased from Hyclone Laboratories Inc. (Logan, UT, USA). Trypsin/

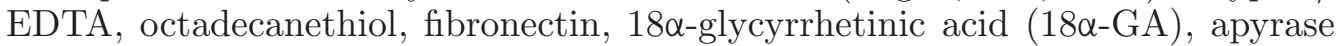
and thapsigargin were ordered from Sigma-Aldrich Co. (St Louis, MO, USA). The fluorescent calcium indicator Fluo-4/AM was obtained from Molecular Probes (Carlsbad, CA, USA).

\section{(b) Bone cell network}

To resemble the in vivo condition for bone cells and normalize the extracellular environment for each individual cell, micro-contact printing and SAM chemistry technologies were employed to build the in vitro cell networks for mechanotransduction experiments (Singhvi et al. 1994; Guo et al. 2006). To investigate the major mechanism of the intercellular calcium signal propagation between bone cells, a linear cell chain pattern and a looped hexagonal cell chain pattern were designed (figure 1). The diameter of the round island for a cell to reside in was $20 \mu \mathrm{m}$. The edge-to-edge distance between neighbouring islands was $70 \mu \mathrm{m}$. The neighbouring islands were connected with $2 \mu \mathrm{m}$ wide connection lines, through which the two neighbouring cells were able to build gap junctions. Two extra short branches were added on each island to provide a physiologically mimicked area for a cell to attach. These geometric parameters were specifically optimized for osteoblast-like MC3T3-E1 cells (Guo et al. 2006). In a linear chain pattern, four cells were connected into a straight line with equal intervals. In a looped pattern, six cells were connected into a hexagon. These designed patterns were first printed on a chromium mask, and then the pattern was replicated to a 


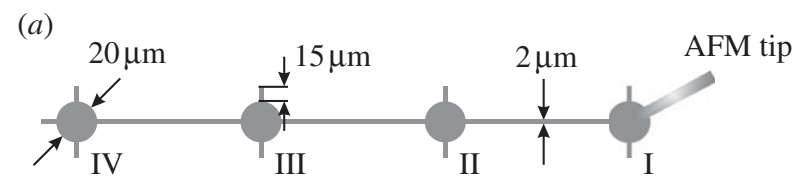

(b)

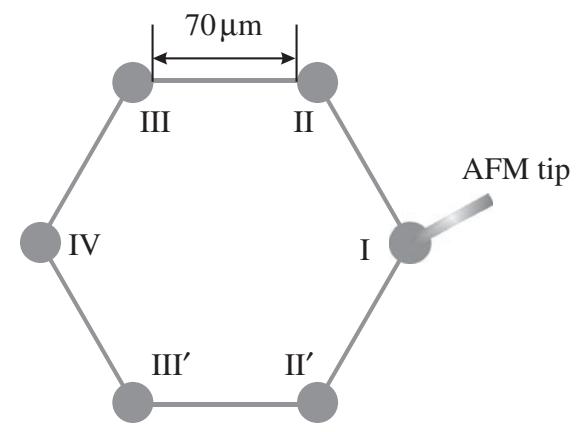

Figure 1. Schematic drawings of the designed (a) linear and (b) looped hexagonal chain patterns. One of the cells in the cell chain is mechanically stimulated by indenting on it with an atomic force microscope (AFM) tip.

master made of positive photo-resist (Shipley 1818, MicroChem Corp., Newton, MA, USA) by exposing the master to UV light through the chromium mask. Using the developed silicon master, a micro-contact printing stamp was fabricated by pouring a 10:1 weight ratio mixture of polydimethylsiloxane (PDMS) and curing agent (Dow Corning, Midland, MI, USA) onto the master template, followed by curing at $70^{\circ} \mathrm{C}$ for $2 \mathrm{~h}$. Finally a micro-contact printing stamp with the designed pattern was obtained by cutting and lifting the PDMS off the master surface.

MC3T3-E1 osteoblastic cells were cultured in $\alpha$-MEM containing 10 per cent FBS and 1 per cent $\mathrm{P} / \mathrm{S}$ and maintained at $37^{\circ} \mathrm{C}$ and 5 per cent $\mathrm{CO}_{2}$ in a humidified incubator. To construct a bone cell network with the designed patterns, the PDMS stamp was inked with a saturated ethanolic solution of octadecanethiol, an adhesive SAM and then stamped on a custom gold-coated glass slide (E-beam evaporator; SC2000, SEMICORE Inc., Livermore, CA, USA). To create protein-resistant regions, the stamped glass slide was immediately immersed in a non-adhesive ethylene glycol-terminated SAM solution (HS-C11$\mathrm{EG}_{3}$; Prochimia, Sopot, Poland) for at least $3 \mathrm{~h}$. A monolayer of $\mathrm{EG}_{3}$, which can effectively resist protein absorption and cell adhesion, was formed on areas that were not stamped with the adhesive SAM. To further facilitate the cell adhesion on the stamped regions, the glass slide was incubated in a fibronectin solution $\left(10 \mu \mathrm{g} \mathrm{ml}^{-1}\right)$ for $1 \mathrm{~h}$. Finally, osteoblast-like MC3T3-E1 cells were dropped onto the glass slide $\left(1 \times 10^{4} \mathrm{cell} \mathrm{cm}^{-2}\right.$ slide area $)$ for seeding and gently washed after an hour to remove unattached cells. The cell-seeded glass slide was cultured in $\alpha$-MEM with 2 per cent charcoal-stripped FBS for at least $24 \mathrm{~h}$ before further experimentation. In a well-constructed cell network (figures 2 and 3), each round island was occupied by a cell, and each cell was physically connected with its neighbouring cells. 

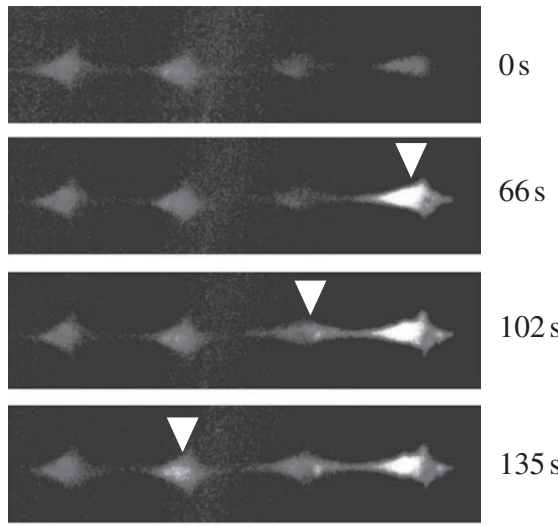

$135 \mathrm{~s}$

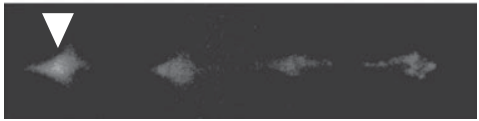

$369 \mathrm{~s}$

Figure 2. Fluorescent images in pseudo-colour of a typical intercellular calcium signal propagation process in a linear bone cell chain. The cell at the right end has been mechanically stimulated by an atomic force microscope probe from $60 \mathrm{~s}$. Its $\left[\mathrm{Ca}^{2+}\right]_{\mathrm{i}}$ intensity reached the peak $6 \mathrm{~s}$ later. The calcium signalling was later transferred to the other cells on its left side, and all three cells released calcium spikes sequentially. The white arrowheads highlight the cell with peak $\left[\mathrm{Ca}^{2+}\right]_{\mathrm{i}}$ at the image-taken time, which is shown on the right.

\section{(c) Atomic force microscope indentation and intracellular calcium responses}

To record the $\left[\mathrm{Ca}^{2+}\right]_{\mathrm{i}}$ transient under mechanical stimulation, the cell networks were dyed with $5 \mu \mathrm{M}$ Fluo-4 AM (Molecular Probes, Eugene, OR, USA) for $1 \mathrm{~h}$ in the incubator and then washed gently with fresh medium three times to remove the extra dye. After Fluo-4 loading, the glass slide with cell networks was cultured in a Petri dish and placed on an atomic force microscope (AFM; Bioscope, Digital Instruments/Veeco, Santa Barbara, CA, USA). The position of the Petri dish was fixed with air vacuum. The AFM was mounted on top of an inverted fluorescence microscope (Olympus IX-70). A 20 min resting period was allowed for the cell network to equilibrate to ambient conditions before further nano-indentation. A 20 min resting was shown to be sufficient for bone cells to recover from a mechanical stimulation and to regenerate repetitive $\left[\mathrm{Ca}^{2+}\right]_{\mathrm{i}}$ responses (Godin et al. 2007). During the experiment, a single cell at one end of the linear chain pattern or at one side of the hexagonal chain pattern was mechanically stimulated by continuous indenting on the cell surface at $1 \mathrm{~Hz}$ frequency for $300 \mathrm{~s}$ with a micro-probe (Hantschel et al. 2002). The magnitude of the indenting force was $60 \mathrm{nN}$. A dedicated study, which compared the effects of loading with different magnitudes, showed that $60 \mathrm{nN}$ force can induce efficient calcium responses in osteoblasts without damaging the plasma membrane (results not shown). Fluorescence images of the cell network were taken every $3 \mathrm{~s}$ to record the cell calcium responses using a cooled digital charged-coupled device (CCD) camera (Sensicam, Cooke Corp., Auburn Hills, MI, USA) during the entire experiment. Cell images in one minute before stimulation were also taken as the baseline for later analysis. 
$0 \mathrm{~s}$

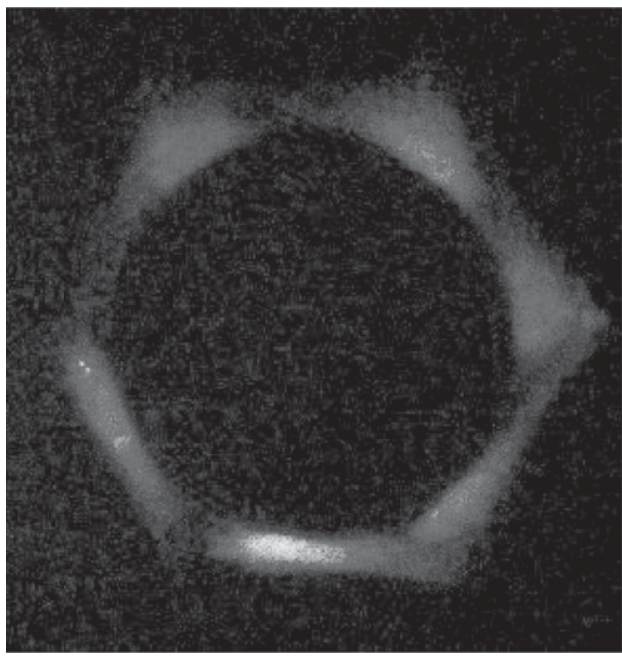

$98 \mathrm{~s}$

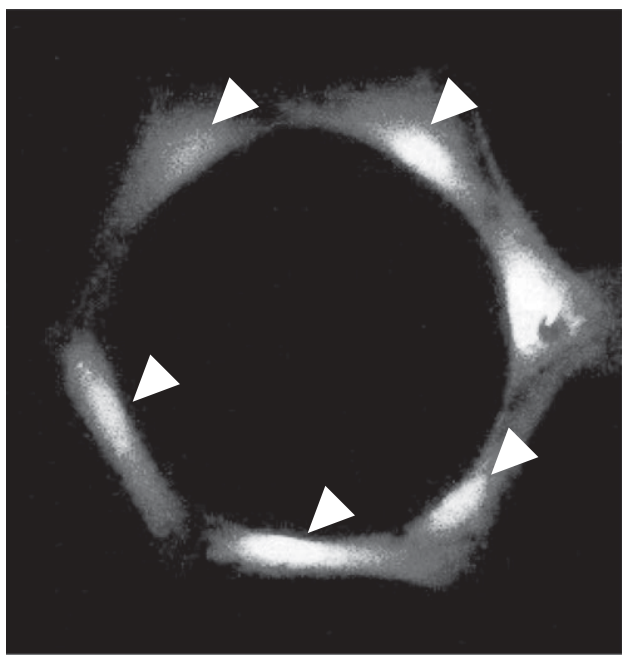

$68 \mathrm{~s}$

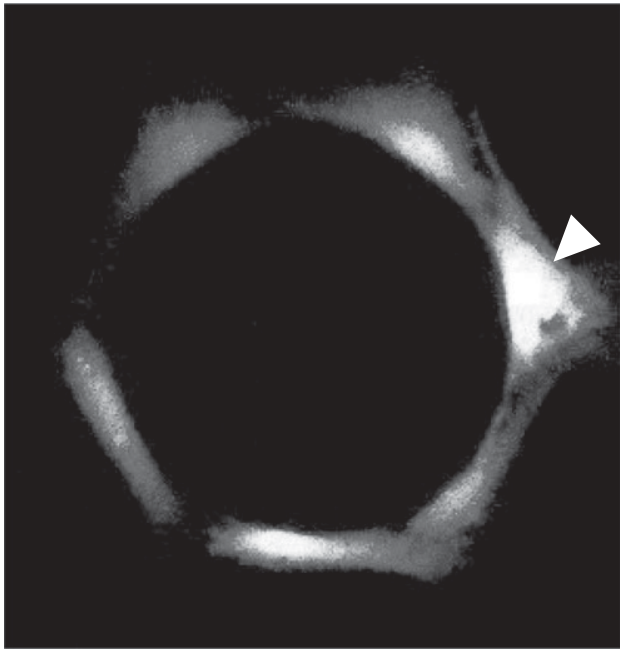

$282 \mathrm{~s}$

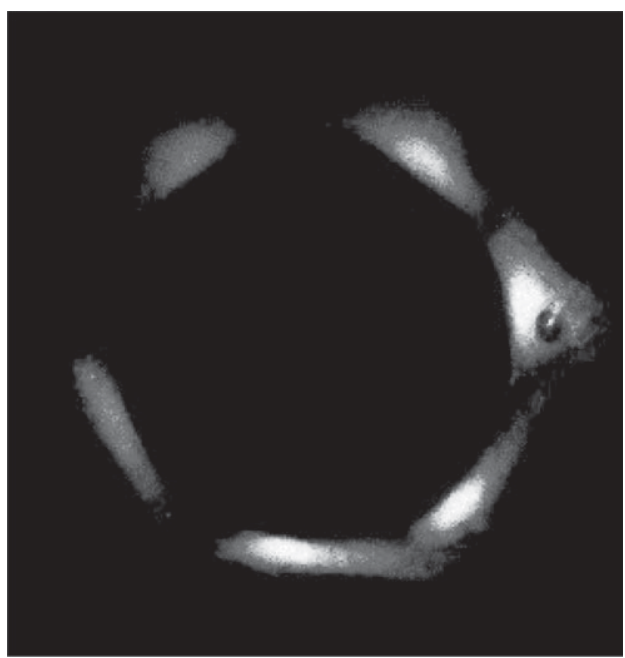

Figure 3. Fluorescent images in pseudo-colour of a typical intercellular calcium signal propagation process in a looped hexagonal bone cell chain. The cell on the right side has been mechanically stimulated by an AFM indentation probe from $54 \mathrm{~s}$. The $\left[\mathrm{Ca}^{2+}\right]_{\mathrm{i}}$ intensity of the indented cell reached the peak $14 \mathrm{~s}$ later. The calcium signal was later transferred to the other cells in the looped chain in both left and right directions. The white arrowheads highlight the cells with peak $\left[\mathrm{Ca}^{2+}\right]_{\mathrm{i}}$ at the image-taken time, which is shown on the top of each image.

\section{(d) Pharmacological agents and experimental groups}

To investigate the mechanism of intercellular calcium propagation and interpret the differences between the results from linear and hexagonal patterns, the linear chain cell networks were treated with different pharmacological agents that blocked specific pathways involved in calcium signalling. Besides the untreated 
group (44 samples for one-dimensional linear pattern), seven groups of chemical agents were employed to treat the cells before/during experiments.

- Vehicle DMSO control. Two of the employed chemicals were dissolved in DMSO. Vehicle control testing was performed with 0.3 per cent v/v DMSO in medium during experiments (six samples).

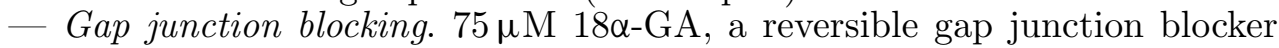
which binds to membrane proteins and causes disassembly of gap junction plaques (Guo et al. 1999), was supplied in Fluo-4 AM dye medium and remained in medium during experiments (Yellowley et al. 2000; 12 samples).

- Extracellular ATP blocking. $10 \mathrm{U} \mathrm{ml}^{-1}$ apyrase, an enzyme that can rapidly hydrolyse extracellular nucleotides, was supplied in the medium $30 \mathrm{~min}$ before and during the experiments (Riddle et al. 2007; 18 samples).

- Gap junction and ATP blocking. Both $75 \mu \mathrm{M} 18 \alpha-\mathrm{GA}$ and $10 \mathrm{Uml}^{-1}$ apyrase were added in the medium $30 \mathrm{~min}$ before experiments to simultaneously block the signal transfer through gap junctions and the diffusion of extracellular ATP (five samples).

- Depletion of intracellular calcium store in ER. $1 \mu \mathrm{M}$ thapsigargin, which can deplete the calcium inside the ER store, was added to the medium 30 min before the mechanical stimulation experiment (Hung et al. 1997; seven samples).

- Depletion of extracellular calcium. Calcium-free DMEM and calcium-free HBSS were used during the mechanical stimulation experiment to remove the extracellular calcium sources (19 samples).

- Depletion of both intracellular calcium store and extracellular calcium resource. After dying with Fluo-4, the cell-seeded slides were first incubated in $1 \mu \mathrm{M}$ thapsigargin for $30 \mathrm{~min}$, and the calcium-free DMEM was used through the experiment (six samples).

Selection of the above agents and the final employed concentrations were determined based on the cited literature in bone cell-related studies. These agents have been demonstrated to be effective in inhibiting the corresponding pathways in bone cells.

\section{(e) Data analysis}

The recorded images were analysed using METAMORPH imaging software (Molecular Devices, Downingtown, PA, USA). The $\left[\mathrm{Ca}^{2+}\right]_{\mathrm{i}}$ transient of each cell was obtained by measuring the average image intensity of the cell in each timelapse image. Individual cells in a cell network were denoted with a Roman number according to its distance from the mechanically indented cell, which itself is numbered as cell I. The cell one step away was denoted as cell II and so on (figure 1). A cell was defined as responsive to the mechanical stimulation if it successfully released a calcium spike with a magnitude four times higher than its own maximum oscillation during the period of baseline measurement (Donahue et al. 2003). The responsive percentage of the cell at a specific location, e.g.

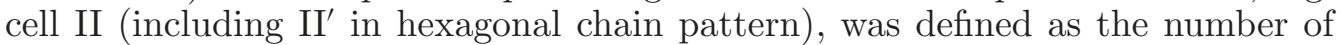
responded cell II divided by the total number of cell II in the experimental group. For a linear chain pattern, the number of cell II or cell III is equal to the number of tested samples. For the hexagonal chain pattern, however, the total number of 
cell II, including both II and II', is twice the sample number, and the same for cell III. The number of responsive cells in a cell network was defined as the total number of responsive cells minus one (the indented cell). Therefore, a count of zero occurs if there are no responsive neighbouring cells except the indented cell, and as one if the signal only transferred to the cell one step away (no responses from cell III or cell IV) in the linear pattern. To evaluate the decay speed of the $\left[\mathrm{Ca}^{2+}\right]_{\mathrm{i}}$ spike, the relaxation part of the $\left[\mathrm{Ca}^{2+}\right]_{\mathrm{i}}$ response curve was fitted with the equation $y=y_{0}+A \mathrm{e}^{-t / \tau}$, and the lifetime constant, $\tau$, was obtained and compared between linear and hexagonal networks.

One-way analysis of variance (ANOVA) with Bonferroni's post hoc analysis was performed to determine statistical differences between mean values of different treatments/groups. Statistical significance is indicated by asterisks $\left({ }^{*} p<0.05\right)$. The condition of constant population variation of treatments was tested by Levene's method.

\section{Results}

\section{(a) Typical calcium responses}

When a cell was indented by an AFM probe, a strong $\left[\mathrm{Ca}^{2+}\right]_{\mathrm{i}}$ spike was released in response to the stimulation, and the cell body turned much brighter in the fluorescence image (figure 2). In most tested samples, this indentation-induced $\left[\mathrm{Ca}^{2+}\right]_{\mathrm{i}}$ response was observed to be successfully transferred to the other cells in the network. Fluorescence images in figure 2 demonstrate a typical intercellular calcium signal transfer process in a linear cell pattern. The cell at the right end, cell I, was mechanically stimulated at $60 \mathrm{~s}$, and the $\left[\mathrm{Ca}^{2+}\right]_{\mathrm{i}}$ intensity of the cell reached a peak in $6 \mathrm{~s}$ (figure 2). The calcium signalling was further transferred to the left cell, cell II, and triggered a calcium transient which reached its peak $36 \mathrm{~s}$ later after that of cell I. Later, the calcium signalling was sequentially transferred to cell III and cell IV. In this particular case, calcium signal transfer from cell III to cell IV took approximately $200 \mathrm{~s}$, although it is rare to have the signal transfer to cell IV in a linear pattern. In the looped hexagonal pattern, the calcium signal was transferred from the indented cell to the other cells in both left and right directions (figure 3). In this particular case shown in figure 3, cell II and II' turned brighter almost simultaneously, so did cell III and III'. In most tests, the calcium signal was successfully transferred to cell IV in the looped network. For both linear and looped patterns, according to the brightness of the cell body, the indented cell always released a $\left[\mathrm{Ca}^{2+}\right]_{\mathrm{i}}$ spike with the highest magnitude in its network. Figure 4 shows a set of typical $\left[\mathrm{Ca}^{2+}\right]_{\mathrm{i}}$ transient curves from both patterns. The $\left[\mathrm{Ca}^{2+}\right]_{\mathrm{i}}$ concentration of the indented cell increased sharply and reached the peak in approximately $10 \mathrm{~s}$ after mechanical stimulation. The other cells in the same network released $\left[\mathrm{Ca}^{2+}\right]_{\mathrm{i}}$ spikes sequentially with much lower magnitudes.

\section{(b) Transfer of calcium signalling}

The transfer efficiencies of calcium signalling in the linear and hexagonal cell networks are summarized and compared in figure 5. In the linear pattern group, the calcium signalling from the indented cell was transferred and successfully 

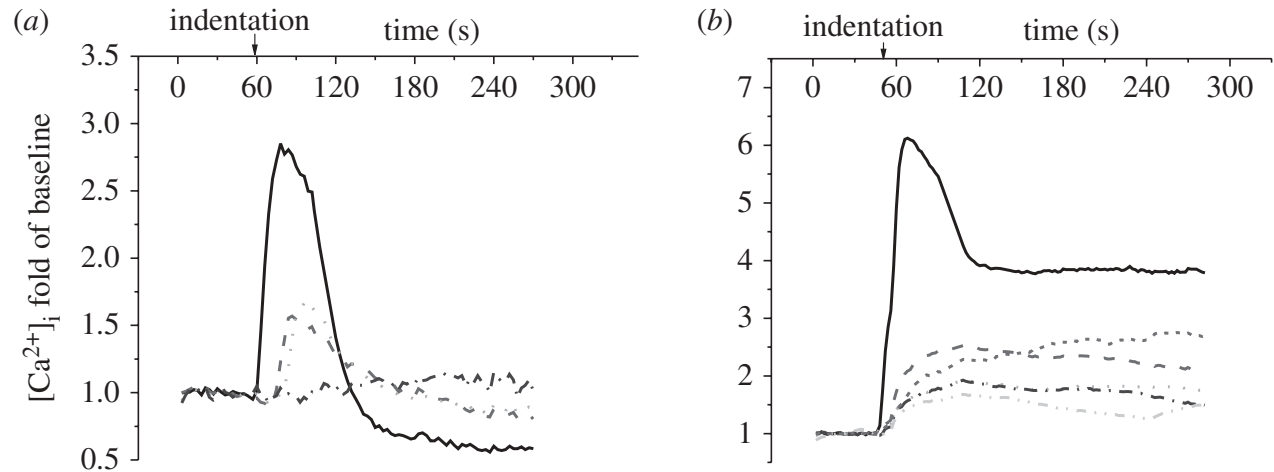

Figure 4. A set of typical $\left[\mathrm{Ca}^{2+}\right]_{\mathrm{i}}$ transients of individual cells in the $(a)$ linear and $(b)$ hexagonal chain patterns. Roman number represents the position of a cell in the network as defined in figure 1 . The calcium responses were recorded for approximately $5 \mathrm{~min}, 1 \mathrm{~min}$ for baseline and 4 min during AFM indentation. The $\left[\mathrm{Ca}^{2+}\right]_{\mathrm{i}}$ concentration is represented by the cell image intensity normalized to the average value of baseline images. Solid lines, cell I; large dashed lines, cell II; small dashed lines, cell II'; dotted lines, cell III; dashed double dotted lines, cell III'; dashed dotted lines, cell IV.

(a)

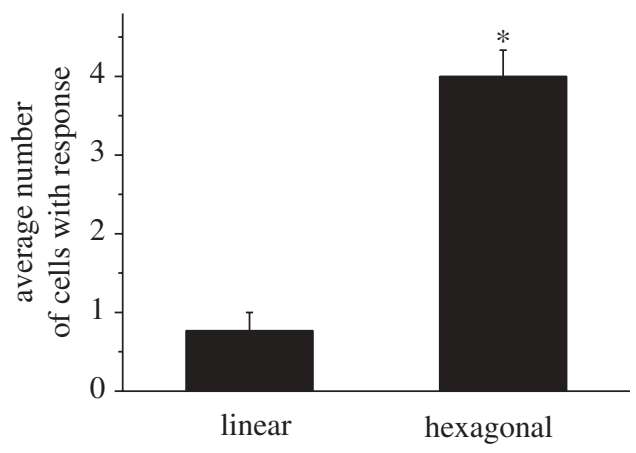

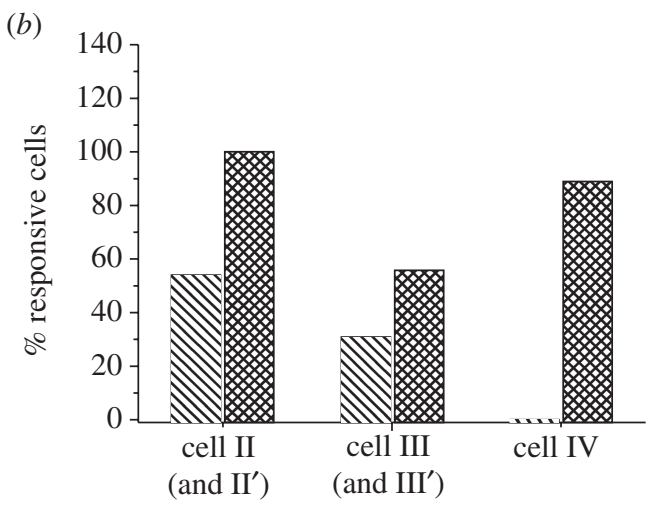

Figure 5. Transferred distance of calcium signalling in the linear (untreated group) and hexagonal bone cell networks. (a) Average number of cells that showed calcium responses after indentation stimulation on a cell in the network (itself excluded) and (b) percentage of cells that showed calcium responses at different positions. The error bars are s.e.m., while the asterisk indicates statistical significance of $p<0.05$ with linear pattern. Bars with cross lines indicate linear pattern; bars with squared lines indicate hexagonal pattern.

induced responses in $0.77 \pm 0.23$ (mean \pm s.e.m.) cells. This number increased almost four times $(4.00 \pm 0.33)$ when the cells were connected in a hexagonal loop. The responsive rate of cells at each individual position was further calculated (figure $5 b$ ). In linear cell networks, the responsive rate of cells decreased as their distances to the indented cell increased. Fifty-four per cent of cell II showed calcium responses, whereas 31 per cent of cell III and no cell IV responded. In the looped hexagonal pattern, the responsive rates of cells at all positions were higher than those in the linear pattern. The responsive rate at position II, 
(a)

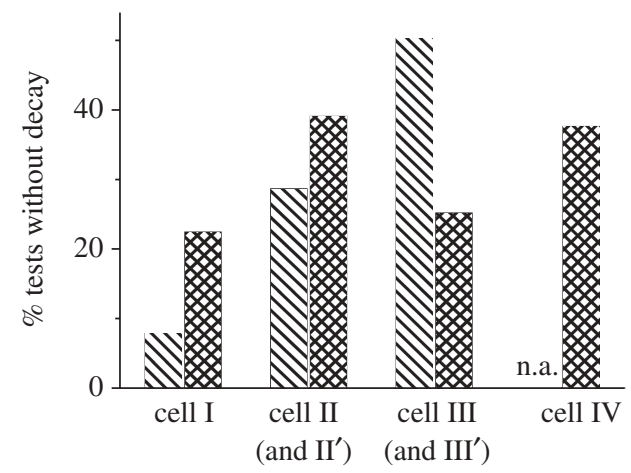

(b)

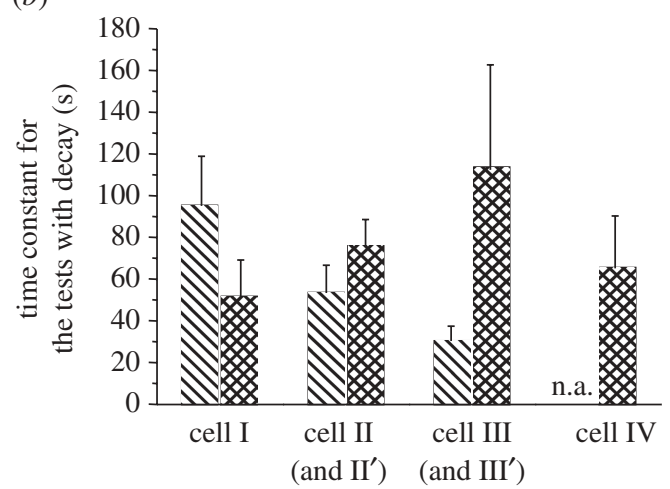

Figure 6. The decay of $\left[\mathrm{Ca}^{2+}\right]_{\mathrm{i}}$ intensity during measurement for linear (untreated group) and hexagonal bone cell networks. (a) Percentage of the tests without decay. (b) The life time constant, $\tau$, obtained by curve-fitting the $\left[\mathrm{Ca}^{2+}\right]_{\mathrm{i}}$ transient with the function $y=y_{0}+A \mathrm{e}^{-t / \tau}$. No significant difference was detected between linear and hexagonal patterns. Bars with cross lines indicate linear pattern; bars with squared lines indicate hexagonal pattern.

including both cell II and $\mathrm{II}^{\prime}$, was as high as 100 per cent, and that at position III was 56 per cent. Interestingly, 89 per cent of cell IV in the hexagonal pattern showed calcium responses, which is even higher than that at position III.

Although the mechanical stimulation was applied on cell I throughout the entire experiment, the cell's $\left[\mathrm{Ca}^{2+}\right]_{\mathrm{i}}$ intensity decreased slowly after reaching the first peak (figure 4). However, a portion of the cells, especially the indirectly stimulated cells, released a calcium spike without decay. After the initial increase, the $\left[\mathrm{Ca}^{2+}\right]_{\mathrm{i}}$ concentration inside the cells reached a plateau without decreasing to its original level. In linear cell networks, 8 per cent for cell I, 29 per cent for cell II and 50 per cent for cell III released calcium spikes without decay. Since very few tests had calcium signal transfer to three cells away in the linear network, this number is not available for cell IV. For the responded cells with decay, a life time constant, $\tau$, was obtained by curve fitting the $\left[\mathrm{Ca}^{2+}\right]_{\mathrm{i}}$ transient curve with a standard exponential function. The time constant ranged between 20 and $120 \mathrm{~s}$ and decreased as distance from the indented cell increased (figure 6b), although no significant difference was detected between cells at different positions. In the looped hexagonal pattern, 22 per cent of cell I showed a $\left[\mathrm{Ca}^{2+}\right]_{\mathrm{i}}$ concentration plateau, much higher than the same percentage in the linear pattern. At positions II, III and IV, a significant portion of the cells showed calcium responses without decay (39\% at position II, $25 \%$ at position III, and $38 \%$ at position IV). The relaxation lifetime for the cells with decay varied from 40 to $140 \mathrm{~s}$. Again, no statistical difference was detected between cells at different positions, or between the linear and hexagonal patterns.

\section{(c) Effects of pharmacological agents}

To investigate the mechanism of intercellular calcium signal propagation, the linear cell networks were divided into eight groups, and each group was treated with different pharmacological agents. The $\left[\mathrm{Ca}^{2+}\right]_{\mathrm{i}}$ transients of the indented cell and the three neighbouring cells from the eight groups are plotted in figure 7 . 

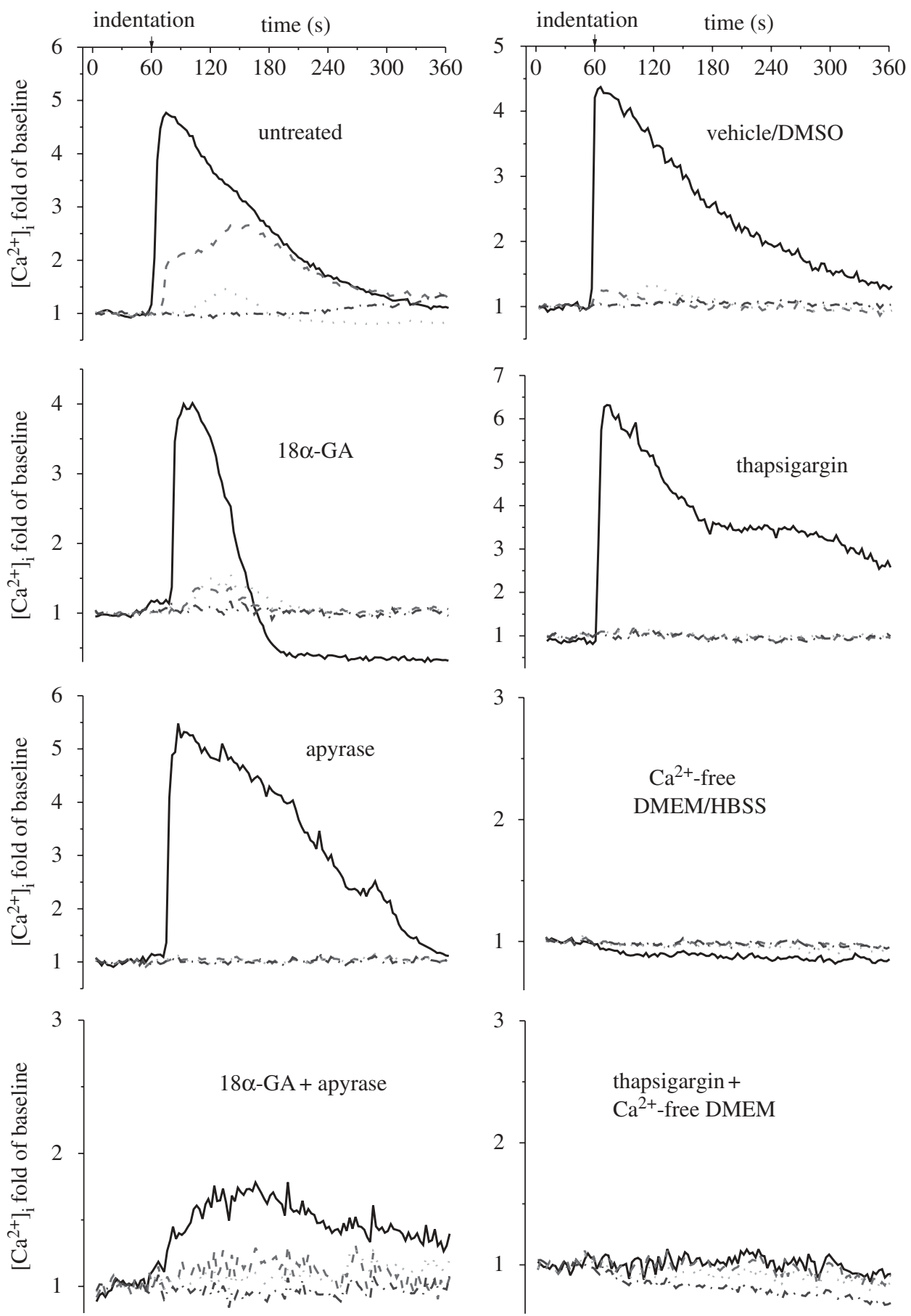

Figure 7. Typical $\left[\mathrm{Ca}^{2+}\right]_{\mathrm{i}}$ transients of each individual cell in the linear bone cell networks for all inhibitor-treated groups. The $\left[\mathrm{Ca}^{2+}\right]_{\mathrm{i}}$ responses of bone cell networks were recorded for $6 \mathrm{~min}, 1 \mathrm{~min}$ for baseline and 5 min during mechanical stimulation. The $\left[\mathrm{Ca}^{2+}\right]_{\mathrm{i}}$ concentration is represented by the cell image intensity normalized to the average value of baseline images. Solid lines, cell I; dashed lines, cell II; dotted lines, cell III; dashed dotted lines, cell IV. 
In most of the tested groups, except the two groups that used calcium-free medium, a robust $\left[\mathrm{Ca}^{2+}\right]_{\mathrm{i}}$ responsive peak in cell I was observed immediately after the start of AFM indentation. The neighbouring cells in the network, cell II, III, and IV, also showed $\left[\mathrm{Ca}^{2+}\right]_{\mathrm{i}}$ waves in the untreated, vehicle control and gap junction-blocked groups. In all groups, the magnitudes of the $\left[\mathrm{Ca}^{2+}\right]_{\mathrm{i}}$ peaks of the neighbouring cells were much lower than those of the indented cells. The intercellular calcium signal transfer was barely observed in the ER calcium store-depleted group and the extracellular ATP hydrolysed group, although the indented cell in these two groups showed normal $\left[\mathrm{Ca}^{2+}\right]_{\mathrm{i}}$ responses. When the cells were treated with both gap junction blocker and ATP inhibitor, only the indented cell showed a weak response. When the extracellular medium was free of calcium, none of the cells in the network, even the directly indented one, was able to generate $\left[\mathrm{Ca}^{2+}\right]_{\mathrm{i}}$ responses to mechanical stimulation.

The average transferred distance of calcium signalling in different groups is shown in figure $8 a$. For the tested samples without signal transfer, i.e. only cell I responded, the transfer number was counted as zero. The average transferred distance of the untreated group was $0.72 \pm 0.19$, similar to the vehicle control and gap junction-blocked groups. No significant difference was detected between these three groups. The hydrolysis of extracellular ATP or depletion of ER calcium store significantly decreased the transfer distance of the calcium signal, $0.33 \pm 0.24$ and $0.11 \pm 0.11$, respectively. When both ATP and gap junctions were blocked or calcium-free medium was used, no transfer was detected in the tested samples. Figure $8 b$ further presents the responsive percentage of cells at different positions. About $30-50 \%$ of cell II showed $\left[\mathrm{Ca}^{2+}\right]_{\mathrm{i}}$ responses in the untreated, vehicle control and gap junction-blocked groups and no significant difference was detected between these three groups. For the other five groups, the responsive rates of cell II shared exactly the same pattern with that of the transferred distance in figure $8 a$. The responsive percentage of cell III was significantly lower than that of cell II. Only 28 per cent of cell III in the untreated group was responsive, which was higher than the vehicle control group (17\%) and gap junction-blocked group (22\%). Nine per cent of cell III in the apyrase-treated group was responsive. The calcium store-depleted group had no responsive cell III that was similar to the other three groups.

\section{Discussion}

This study investigated the major mechanisms, specifically the diffusion of extracellular ATP and intercellular gap junction communication, involved in calcium wave propagation between bone cells. To achieve this goal, two types of two-dimensional cell networks with different geometric layouts were designed and constructed. Although the bone cell networks in vivo are three-dimensional and more complicated than the present two-dimensional networks, the simplicity of the two-dimensional cell pattern endows it with an inherent advantage for studying the intercellular signal propagation. The calcium responses were induced by indentation on a single cell in the network, and the calcium waves in all the cells were recorded, analysed and compared. To further clarify the molecular pathways involved in the signal transfer, a set of pharmacological agents were employed to block certain pathways in the cells. 

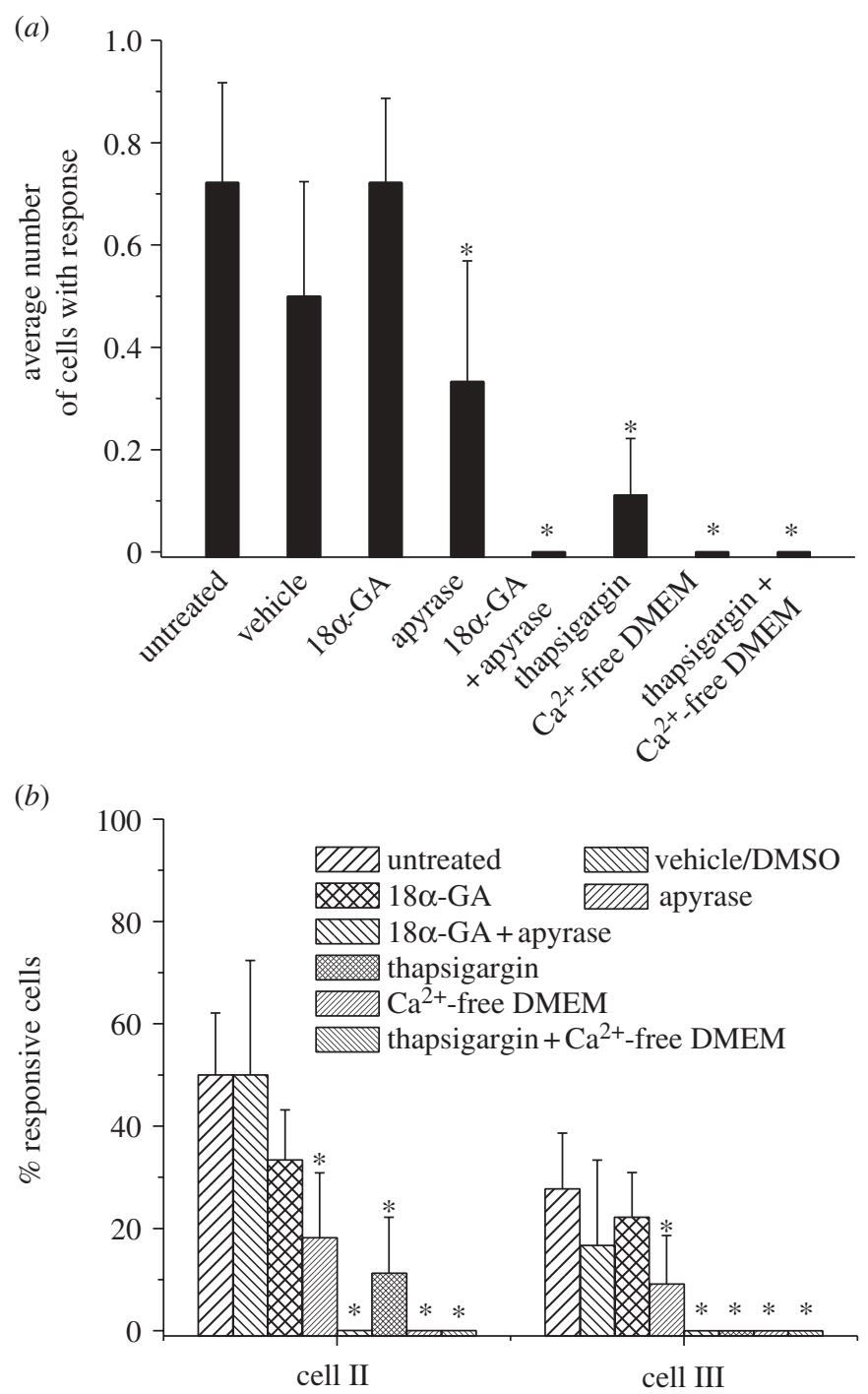

Figure 8. Comparison of $\left[\mathrm{Ca}^{2+}\right]_{\mathrm{i}}$ signal transfer in linear cell networks between all pharmacologically treated groups. (a) The average transferred distance of calcium signalling. (b) Responsive percentage of cell II and III in the cell networks. Cell I was mechanically stimulated with an AFM probe. The error bars are s.e.m.; the asterisks indicate statistical significance of $p<0.05$ with untreated.

The results from pathway-inhibitor studies showed that calcium wave propagation was significantly impeded when extracellular ATP in the medium was hydrolysed by apyrase. Chemical uncoupling of gap junctions, however, did not significantly decrease transferred distance of calcium wave in the network (figure 8). It is, therefore, extracellular ATP diffusion, rather than molecular transport through gap junctions, that dominantly mediates the transmission of mechanically elicited intercellular calcium signalling in bone cells. The calcium wave and responsive rate of cells in the linear and hexagonal patterns further 
supported this conclusion. If gap junction communication is the sole pathway for signal propagation, the second messengers, which can be transferred through the gap junctions and further induce calcium responses, such as $\mathrm{IP}_{3}$ and $\mathrm{Ca}^{2+}$, can only be transferred in one direction from the indented cell in the linear network. In the hexagonal pattern, the messengers would be divided and diffused into both cells on left and right sides in the loop. The number of cells with responses in hexagonal networks should be approximately twice that in linear networks, or lower than twice owing to the partition. However, the results from the present study showed that the responsive percentage of cells in the hexagonal pattern are almost four times those in the linear network. The signal propagation efficiency in the looped network is much higher than that in the linear network. If extracellular ATP diffusion is the major mechanism for signal transfer, this discrepancy would be easily explained. In the looped pattern, all cells are closer to the indented cell. Cell IV, for instance, is only two steps away from cell I instead of three steps away in the linear pattern. This would shorten the signal transfer time and increase the ATP concentration around the cell body.

Another potential mechanism that sustained the high efficiency of signal transfer in the looped network is the sequential release of ATP by cells along the path of the calcium wave. It is difficult to conclude whether there is sequential release of ATP in the network based solely on the results from the linear pattern. The intensity of $\left[\mathrm{Ca}^{2+}\right]_{\mathrm{i}}$ responses dampened as the signalling wave spread from cell to cell. The responsive rate of cells decreased sharply as the distance away from the indented cell increased (figure $5 b$ ). It is more likely the calcium wave in the linear pattern can be described by a point source model. In the looped pattern, however, the responsive rate of cell IV was higher than that of cell III. This conflicts with the assumption that cell I is the only ATP source, as cell III is closer to the source (cell I) than cell IV is. Therefore, there is sequential ATP release, although the ATP release from neighbouring cells is markedly lower than that from the indented cell. In the looped networks, cell IV can receive ATP released from two cells one step away, both cell III and III', whereas cell III or III' itself has only one direct upstream cell. This mechanism may partially explain the higher response rate of cell IV, although the confirmation of the existence of sequential ATP release in neighbouring cells may require further studies. In the present study, a portion of the cells, especially the non-indented cells, showed a calcium spike without decay. This has been reported in a similar study using smooth muscle cells (Fanchaouy et al. 2005). Thirty-five per cent of the muscle cells released sustained calcium responses under ATP stimulation. The cells maintained elevated $\left[\mathrm{Ca}^{2+}\right]_{\mathrm{i}}$ for more than $6 \mathrm{~min}$. These findings are highly consistent with the present study, although the exact mechanism is yet unclear.

In bone cells, both mechanical stimulation and activation of purinergic receptors can result in the activation of PLC and generation of $\mathrm{IP}_{3} . \mathrm{IP}_{3}$ binds to and activates the $\mathrm{InsP}_{3}$ receptor on the membrane of the ER, opening a calcium channel, resulting in the release of calcium (Berridge 1993; Jorgensen et al. 2000). Since both $\mathrm{IP}_{3}$ and calcium can pass through gap junctions, it has long been conjectured that GJIC is an important mechanism for calcium wave propagation in bone cell networks. In the present study, however, the results from both network comparison and pathway inhibition implied that diffusion of ATP is more important than GJIC. Previous studies also showed that GJIC in MC3T3-E1 
cells does not allow passage of $\mathrm{IP}_{3}$ well enough to elicit the release of intracellular calcium stores to the neighbouring cells. The $\left[\mathrm{Ca}^{2+}\right]_{\mathrm{i}}$ response was unaltered by the disruption in gap-junctional communication (Saunders et al. 2001, 2003). Although the present results demonstrate a significant role for ATP in MC3T3E1 calcium wave propagation, our findings did not rule out the involvement of $\mathrm{IP}_{3}$ or calcium that can diffuse through gap junction pores. The results showed that treatment with apyrase alone can significantly reduce, but not completely abolish calcium signal propagation. When $18 \alpha-$ GA was employed together with apyrase for cell treatment, the intercellular calcium wave was abolished. Treatment with $18 \alpha-G A$ alone, however, had no significant effect on calcium wave propagation. Therefore, the present results implied that transfer of second messengers through gap junction pores still existed and slightly assisted the propagation of calcium waves in the MC3T3-E1 cell network, although this could be easily overshadowed by the purinergic receptor pathway.

Current experimental results from calcium-free media and ER calcium store depletion groups have identified the calcium sources underlying the $\left[\mathrm{Ca}^{2+}\right]_{\mathrm{i}}$ responses in MC3T3-E1 cells. Cells in calcium-free medium showed no response to either fluid flow or AFM indentation stimulations. This implies that the $\left[\mathrm{Ca}^{2+}\right]_{\mathrm{i}}$ transients in MC3T3-E1 cells induced by mechanical stimulation have to be initiated with extracellular calcium influx. Another major calcium source for $\left[\mathrm{Ca}^{2+}\right]_{\mathrm{i}}$ responses is the intracellular calcium store, which is mainly held within the membrane systems of the ER (Berridge 1993; Clapham 1995). In this study, depletion of the ER stores with thapsigargin severely decreased intercellular calcium signalling, although the indented cell showed normal responses. As discussed previously, the ATP-elicited $\left[\mathrm{Ca}^{2+}\right]_{\mathrm{i}}$ responses in the neighbouring cells are mainly generated by $\mathrm{IP}_{3}$ and $\mathrm{IP}_{3}$-induced calcium release from ER store. When the ER was depleted of calcium, the $\left[\mathrm{Ca}^{2+}\right]_{\mathrm{i}}$ fluctuation was not observed in the cells. The $\left[\mathrm{Ca}^{2+}\right]_{\mathrm{i}}$ transient in cell I was mainly generated by calcium influx owing to mechanical stimulation.

In summary, this study clarified the major mechanism for intercellular calcium wave propagation in osteoblastic networks. It also provided important knowledge about the calcium sources of the $\left[\mathrm{Ca}^{2+}\right]_{\mathrm{i}}$ responses induced by mechanical or ATP stimulations.

This work was supported by NIH grant R21 AR052417 (X. Edward Guo). The authors would like to thank Dr Eugene M. Chow of Palo Alto Research Center for manufacturing the AFM indentation probe. We would also like to thank Mr Andrew D. Baik for editing the manuscript.

\section{References}

Allori, A. C., Sailon, A. M., Pan, J. H. \& Warren, S. M. 2008 Biological basis of bone formation, remodeling, and repair-part III: biomechanical forces. Tissue Eng. Part B Rev. 14, 285-293. (doi:10.1089/ten.teb.2008.0084)

Batra, N. N., Li, Y. J., Yellowley, C. E., You, L., Malone, A. M., Kim, C. H. \& Jacobs, C. R. 2005 Effects of short-term recovery periods on fluid-induced signaling in osteoblastic cells. J. Biomech. 38, 1909-1917. (doi:10.1016/j.jbiomech.2004.08.009)

Berridge, M. J. 1993 Inositol trisphosphate and calcium signalling. Nature 361, 315-325. (doi:10.1038/361315a0)

Berridge, M. J., Lipp, P. \& Bootman, M. D. 2000 The versatility and universality of calcium signalling. Nat. Rev. Mol. Cell Biol. 1, 11-21. (doi:10.1038/35036035)

Phil. Trans. R. Soc. A (2010) 
Carafoli, E. 2002 Calcium signaling: a tale for all seasons. Proc. Natl Acad. Sci. USA 99, 1115-1122. (doi:10.1073/pnas.032427999)

Clapham, D. E. 1995 Calcium signaling. Cell 80, 259-268. (doi:10.1016/0092-8674(95)90408-5)

Donahue, H. J., McLeod, K. J., Rubin, C. T., Andersen, J., Grine, E. A., Hertzberg, E. L. \& Brink, P. R. 1995 Cell-to-cell communication in osteoblastic networks: cell line-dependent hormonal regulation of gap junction function. J. Bone Miner. Res. 10, 881-889.

Donahue, S. W., Donahue, H. J. \& Jacobs, C. R. 2003 Osteoblastic cells have refractory periods for fluid-flow-induced intracellular calcium oscillations for short bouts of flow and display multiple low-magnitude oscillations during long-term flow. J. Biomech. 36, 35-43. (doi:10.1016/ S0021-9290(02)00318-4)

Duncan, R. L. \& Turner, C. H. 1995 Mechanotransduction and the functional response of bone to mechanical strain. Calcif. Tissue Int. 57, 344-358. (doi:10.1007/BF00302070)

Fanchaouy, M., Serir, K., Meister, J. J., Beny, J. L. \& Bychkov, R. 2005 Intercellular communication: role of gap junctions in establishing the pattern of ATP-elicited $\mathrm{Ca}^{2+}$ oscillations and $\mathrm{Ca}^{2+}$-dependent currents in freshly isolated aortic smooth muscle cells. Cell Calcium 37, 25-34. (doi:10.1016/j.ceca.2004.06.008)

Godin, L. M., Suzuki, S., Jacobs, C. R., Donahue, H. J. \& Donahue, S. W. 2007 Mechanically induced intracellular calcium waves in osteoblasts demonstrate calcium fingerprints in bone cell mechanotransduction. Biomech. Model. Mechanobiol. 6, 391-398. (doi:10.1007/s10237006-0059-5)

Guo, Y., Martinez-Williams, C., Gilbert, K. A. \& Rannels, D. E. 1999 Inhibition of gap junction communication in alveolar epithelial cells by $18 \alpha$-glycyrrhetinic acid. Am. J. Physiol. 276, L1018-L1026.

Guo, X. E. et al. 2006 Intracellular calcium waves in bone cell networks under single cell nanoindentation. Mol. Cell. Biomech. 3, 95-107.

Guthrie, P. B., Knappenberger, J., Segal, M., Bennett, M. V., Charles, A. C. \& Kater, S. B. 1999 ATP released from astrocytes mediates glial calcium waves. J. Neurosci. 19, 520-528.

Hantschel, T., Chow, E. M., Rudolph, D. \& Fork, D. K. 2002 Stressed metal probes for atomic force microscopy. Appl. Phys. Lett. 81, 3070-3072. (doi:10.1063/1.1514830)

Hung, C. T., Pollack, S. R., Reilly, T. M. \& Brighton, C. T. 1995 Real-time calcium response of cultured bone cells to fluid flow. Clin. Orthop. Relat. Res. 256-269.

Hung, C. T., Allen, F. D., Mansfield, K. D. \& Shapiro, I. M. 1997 Extracellular ATP modulates $\left[\mathrm{Ca}^{2+}\right]_{\mathrm{i}}$ in retinoic acid-treated embryonic chondrocytes. Am. J. Physiol. 272, C1611-C1617.

Huo, B., Lu, X. L., Hung, C. T., Costa, K. D., Xu, Q. B., Whitesides, G. M. \& Guo, X. E. 2008 Fluid flow induced calcium response in bone cell network. Cell. Mol. Bioeng. 1, 58-66. (doi:10.1007/ s12195-008-0011-0)

Iqbal, J. \& Zaidi, M. 2005 Molecular regulation of mechanotransduction. Biochem. Biophys. Res. Commun. 328, 751-755. (doi:10.1016/j.bbrc.2004.12.087)

Jorgensen, N. R., Geist, S. T., Civitelli, R. \& Steinberg, T. H. 1997 ATP- and gap junctiondependent intercellular calcium signaling in osteoblastic cells. J. Cell Biol. 139, 497-506. (doi:10.1083/jcb.139.2.497)

Jorgensen, N. R., Henriksen, Z., Brot, C., Eriksen, E. F., Sorensen, O. H., Civitelli, R. \& Steinberg, T. H. 2000 Human osteoblastic cells propagate intercellular calcium signals by two different mechanisms. J. Bone Miner. Res. 15, 1024-1032. (doi:10.1359/jbmr.2000.15.6.1024)

Kuroda, Y., Hisatsune, C., Nakamura, T., Matsuo, K. \& Mikoshiba, K. 2008 Osteoblasts induce $\mathrm{Ca}^{2+}$ oscillation-independent NFATc1 activation during osteoclastogenesis. Proc. Natl Acad. Sci. USA 105, 8643-8648. (doi:10.1073/pnas.0800642105)

Riddle, R. C., Taylor, A. F., Rogers, J. R. \& Donahue, H. J. 2007 ATP release mediates fluid flowinduced proliferation of human bone marrow stromal cells. J. Bone Miner. Res. 22, 589-600. (doi:10.1359/jbmr.070113)

Rubin, J., Rubin, C. \& Jacobs, C. R. 2006 Molecular pathways mediating mechanical signaling in bone. Gene 367, 1-16. (doi:10.1016/j.gene.2005.10.028)

Saunders, M. M., You, J., Trosko, J. E., Yamasaki, H., Li, Z., Donahue, H. J. \& Jacobs, C. R. 2001 Gap junctions and fluid flow response in MC3T3-E1 cells. Am. J. Physiol. Cell Physiol. 281, C1917-C1925. 
Saunders, M. M., You, J., Zhou, Z., Li, Z., Yellowley, C. E., Kunze, E. L., Jacobs, C. R. \& Donahue, H. J. 2003 Fluid flow-induced prostaglandin E-2 response of osteoblastic ROS 17/2.8 cells is gap junction-mediated and independent of cytosolic calcium. Bone 32, 350-356. (doi:10.1016/S8756-3282(03)00025-5)

Singhvi, R., Kumar, A., Lopez, G. P., Stephanopoulos, G. N., Wang, D. I., Whitesides, G. M. \& Ingber, D. E. 1994 Engineering cell shape and function. Science 264, 696-698. (doi:10.1126/ science.8171320)

Yao, J., Suwa, M., Li, B., Kawamura, K., Morioka, T. \& Oite, T. 2003 ATP-dependent mechanism for coordination of intercellular $\mathrm{Ca}^{2+}$ signaling and renin secretion in rat juxtaglomerular cells. Circ. Res. 93, 338-345. (doi:10.1161/01.RES.0000086802.21850.5D)

Yellowley, C. E., Li, Z., Zhou, Z., Jacobs, C. R. \& Donahue, H. J. 2000 Functional gap junctions between osteocytic and osteoblastic cells. J. Bone Miner. Res. 15, 209-217. (doi:10.1359/jbmr. 2000.15.2.209)

Zayzafoon, M. 2006 Calcium/calmodulin signaling controls osteoblast growth and differentiation. J. Cell. Biochem. 97, 56-70. (doi:10.1002/jcb.20675) 